of poor quality of shiftwork than predominant forward shift pattern.

Conclusion Even shift work associate with poor quality of sleep but good strategy of shift work scheduling help to decrease risk of poor quality of sleep.

\section{P.2.35 HEART RATE VARIABILITY AND SLEEP-RELATED BREATHING DISORDERS IN COMMERCIAL DRIVERS}

${ }^{1}$ Wei-te Wu*, ${ }^{2} Y u-J e n$ Lin, ${ }^{1}$ Saou-Hsing Liou. ${ }^{1}$ National Institute of Environmental Health Sciences, National Health Research Institutes, Miaoli, Taiwan; ${ }^{2}$ Institute of Occupational Medicine and Industrial Hygiene, National Taiwan University, Taipei, Taiwan

\subsection{6/OEM-2019-EPI.262}

Background Sleep-related breathing disorders, such as obstructive sleep apnea (OSA), seems to have an important influence on the autonomic nervous function on the heart, and associated with cardiovascular mortality. Therefore, we assessed the relations of sleep apnea-related parameters with 5 min heart rate variability (HRV) in a large cohort of male commercial drivers.

Methods This cross-sectional study recruited 245 male bus drivers from one transportation company in Taiwan. Each participant was evaluated by a polysomnography (PSG) test and by blood lipids examination. Severity of OSA was categorized according to the apnea-hypopnea index (AHI). In addition, short-term HRV was tested.

Results Subjects were categorized into severe OSA group $(\mathrm{n}=44 ; 17.9 \%)$, moderate and mild OSA group $(\mathrm{n}=117$; $47.8 \%)$, and non-OSA group $(n=84 ; 34.3 \%)$. AHI had negative correlations with SDNN $(\beta=-0.075, p=0.007)$ and RMSSD $(\beta=-0.121, p<0.001)$, adjusting for age, body mass index, smoking, drinking, systolic blood pressure, HDL, and fasting blood glucose. RMSSD level was decreased with severe OSA $(\beta=-0.266, p=0.042)$ even adjusting for confounders.

Conclusions The findings showed a low level of RMSSD in OSA among commercial drivers, especially in the severe group category. Decreased SDNN and RMSSD levels are associated with AHI level in commercial drivers, suggesting that they are tightly linked with autonomic dysfunction.

\section{P.2.36 ASSESSMENT OF SERUM AND URINARY BIOMARKERS FOR PNEUMOCONIOSIS IN A COHORT OF STONE WORKERS EXPOSED TO ASBESTOS-CONTAMINATED MINERALS}

\begin{abstract}
1,2,3 Hsiao-Yu Yang*, 1,2,3,4 Pau-Chung Chen. 'Institute of Occupational Medicine and Industrial Hygiene, National Taiwan University College of Public Health, Taipei, Taiwan; ${ }^{2}$ Department of Public Health, National Taiwan University College of Public Health, Taipei, Taiwan; ${ }^{3}$ Department of Environmental and Occupational Medicine, National Taiwan University Hospital, Taipei, Taiwan; ${ }^{4}$ Department of Environmental and Occupational Medicine, National Taiwan University College of Medicine, Taipei, Taiwan
\end{abstract}

\subsection{6/OEM-2019-EPI.263}

Background Pneumoconiosis is still a problem in workers process non-asbestiform asbestos minerals and serpentinite rocks, such as nephrite, antigorite or talc that may contaminate with paragenetic asbestos minerals. An effective screening method is still lacking. The objective of this study was to assess the diagnostic accuracy using the serum and urinary biomarkers for pneumoconiosis in workers exposed to asbestos-contaminated minerals.

Methods Prediction models of pneumoconiosis were constructed from 140 stone workers (48 cases of pneumoconiosis and 118 controls) exposed to asbestos-contaminated minerals. We measured serum soluble mesothelin-related peptide (SMRP), fibulin-3, carcinoembryonic antigen, and urinary 8-Oxo-2'-deoxyguanosine (8-OHdG)/creatinine levels. Using the ILO international classification of radiographs of pneumoconiosis profusion subcategory $\geq 1 / 0$ as the reference standard, we established a prediction model by machine learning algorithm. We assessed the accuracy by the area under the receiver operating characteristic curve (AUROC).

Results The SMRP level increased in workers exposed to nephrite. A dose-response relationship was found between the SMRP level and the severity of pneumoconiosis in workers exposed to asbestos-contaminated minerals. Machine learning algorithm composed of sex, age, and 4 serum and urinary biomarkers is able to predict pneumoconiosis with high accuracy (AUROC ranged from 0.76 to 1.00).

Conclusion Our finding highlight the use of serum and urinary biomarkers can be developed as a screening tool for pneumoconiosis in workers exposed to potential asbestos contaminated minerals.

\section{P.2.37 SPANISH FIREFIGHTER'S 10-YEAR FOLLOW-UP STUDY ON CANCER MORTALITY}

${ }^{1}$ Guanlan Zhao*, ${ }^{2}$ Enrique Regidor, ${ }^{1}$ Boris Erazo, ${ }^{1}$ Francisco Brocal, ${ }^{1}$ Elena Ronda. ${ }^{1}$ University of Alicante, Alicante, Spain; ${ }^{2}$ Complutense University of Madrid, Madrid, Spain

\subsection{6/OEM-2019-EPI.264}

Introduction Firefighters are exposed to different occupational risks in their work, some of which are carcinogenic. However, there has no studies on investigating the mortality of this group of workers in Spain. The objective of this study is to compare the mortality rate of all cancer sites in firefighters with the rest of employed workers.

Methods Mortality was calculated from a longitudinal study between 2001-2011. A total of 9.5 million men aged from 20 to 64 years with employment in 2001 were followed. For all cancer sites, the standardized mortality rate (SMR) has been calculated by age per 100000 person per year and the rate ratios (RT) have been estimated for firefighters versus the rest of employed workers with $95 \%$ confidence intervals (95\% CI).

Results The overall mortality of cancer in firefighters is not statistically different from that observed in the rest of the employed workers $(\mathrm{RT}=1.00$ (95\% CI 0.89-1.12)). For most cancer sites, no significant differences were observed in the mortality rates between firefighters versus the rest of employed workers, the exceptions were cancers of the hypopharynx and larynx cancer, which had a higher magnitude in firefighters, whose mortality rate ratios were 2.96 (1.31-6. $69)$ and 1.77 (1.01-3.09), respectively.

Conclusion Exposure to occupational risks factors may be responsible for excess mortality from hypopharyngeal and larynx cancer in firefighters. In the future, more research is needed on the health of firefighters and strengthening preventive policies for these workers. 